\title{
The Hydrodynamic Representation of the Klein-Gordon Equation with Self- Interacting Field
}

Piero Chiarelli *

National Council of Research of Italy, Interdepartmental Center "E. Piaggio" University of Pisa, Area of Pisa, Pisa, Moruzzi 1, Italy

\begin{abstract}
In this paper the quantum hydrodynamic approach for the KGE owing a self-interaction term is developed both for scalar and charged boson. The model allows to determine the quantum energy impulse tensor density of massive bosons such as the mesons. The generalization of the hydrodynamic Klein-Gordon equation to the non-Euclidean space-time is also derived for a quantum relativity approach.
\end{abstract}

Keywords: Quantum hydrodynamic representation; BhomMadelung approach; Self-interacting field; Non-Euclidean quantum hydrodynamics

\section{Introduction}

Since the introduction of the quantum wave equation by Schrödinger, the quantum hydrodynamic approach (QHA) was presented by Madelung [1]. In this quantum representation, developed by Madelung and then by Bhom, the evolution of a complex variable $\psi=|\psi| \exp \frac{i}{\hbar} S$ is solved as a function of the two real variables, $|\psi|$ and $S$ [2-5]. As shown by Weiner et al. [6], the outputs of the quantum hydrodynamic model agree with the outputs of the Schrödinger problem and, more recently, as shown by Koide and Kodama [7], it agrees with the outputs of the stochastic variational method.

Recently, the author has shown that the hydrodynamic approach is strictly correlated to the properties of vacuum on small scale [8].

Moreover, as shown by Bohm and Hiley $[9,10]$ the hydrodynamic approach can be generalized for the description of the quantum fields.

The present work develops the quantum hydrodynamic form of the KleinGordon equation (KGE) containing an additional self-interaction term.

The interest in obtaining such a description lies in the fact that such type of KGE can describe the states of bosons, such as mesons. The goal of the paper is to obtain the energy-impulse tensor density of such particles that can be useful in the coupling the field of a meson with the Einstein equation [11]. The paper is organized as follows: in the section 2 the hydrodynamic KGE with a self-interaction term is derived for an uncharged scalar particle as well as the Lagrangean motion equations for the eigenstates and the associated energy impulse tensor density.. In the subsection 2.2 the theory is developed for a charged field. In section 3. the formulas are generalized to a non-Euclidean space-time.

\section{The Hydrodynamic KGE with Self-Interacting Field}

In this section, the Euclidean hydrodynamic representation of the KGE is derived for a scalar uncharged particle with a self-interaction term that reads

$$
\partial_{\mu} \partial^{\mu} \psi=-\frac{m^{2} c^{2}}{\hbar^{2}} \psi+j_{(\psi)}
$$

where $j_{(\psi)}=-\frac{\partial V_{(\psi)}}{\partial \psi}$ and where, for instance, we assume the quartic renormalizable interaction $V_{(\psi)}=\frac{\lambda}{4} \psi^{* 2} \psi^{2}=\frac{\lambda}{4}|\psi|^{4}$
Following the procedure given in reference [11,12] (for the ordinary KGE) the hydrodynamic equations of motion are given by the Hamilton-Jacobi type equation

$$
g^{\mu \nu} \frac{\partial S_{(q, t)}}{\partial q^{\mu}} \frac{\partial S_{(q, t)}}{\partial q^{v}}-\hbar^{2}\left(\frac{\partial_{\mu} \partial^{\mu}|\psi|}{|\psi|}+\lambda|\psi|^{2}\right)-m^{2} c^{2}=0
$$

coupled to the current equation [2]

$$
\frac{\partial}{\partial q^{\mu}}\left(|\psi|^{2} \frac{\partial S}{\partial q^{\mu}}\right)=m \frac{\partial J_{\mu}}{\partial q^{\mu}}=0
$$

where

$$
\mathrm{S}=\frac{\hbar}{2 \mathrm{i}} \ln \left[\frac{\psi}{\psi^{*}}\right]
$$

and where

$$
\mathrm{J}_{\mu}=\left(\mathrm{c} \rho,-\mathrm{J}_{\mathrm{i}}\right)=\frac{\mathrm{i} \hbar}{2 \mathrm{~m}}\left(\psi^{*} \frac{\partial \psi}{\partial \mathrm{q}^{\mu}}-\psi \frac{\partial \psi^{*}}{\partial \mathrm{q}^{\mu}}\right)
$$

Moreover, being the 4-impulse in the hydrodynamic analogy

$$
\mathrm{p}_{\mu}=\left(\frac{\mathrm{E}}{\mathrm{c}},-\mathrm{p}_{\mathrm{i}}\right)=-\frac{\partial \mathrm{S}}{\partial \mathrm{q}^{\mu}}
$$

it follows that

$$
J_{\mu}=\left(c \rho,-J_{i}\right)=-|\psi|^{2} \frac{p_{\mu}}{m}
$$

where

$$
\rho=\frac{|\psi|^{2}}{\mathrm{mc}^{2}} \frac{\partial \mathrm{S}}{\partial \mathrm{t}}
$$

Moreover, by using (6), equation (2) can be rewritten as

*Corresponding author: Piero Chiarelli, National Council of Research of Italy, Interdepartmental Center "E. Piaggio" University of Pisa, Italy, Tel: +39-050-3152359,Fax: +39-050-315-2166 ; E-mail: pchiare@ifc.cnr.it

Received July 31, 2017; Accepted August 09, 2017; Published August 14, 2017

Citation: Chiarelli P (2017) The Hydrodynamic Representation of the Klein-Gordon Equation with Self-Interacting Field. J Astrophys Aerospace Technol 5: 148. doi:10.4172/2329-6542.1000148

Copyright: @ 2017 Chiarelli P. This is an open-access article distributed under the terms of the Creative Commons Attribution License, which permits unrestricted use, distribution, and reproduction in any medium, provided the original author and source are credited. 


$$
\frac{\partial S}{\partial q^{\mu}} \frac{\partial S}{\partial q_{\mu}}=p_{\mu} p^{\mu}=\left(\frac{E^{2}}{c^{2}}-p^{2}\right)=m^{2} c^{2}\left(1-\frac{V_{q u}}{m c^{2}}\right)
$$

where

$$
V_{q u}=-\frac{\hbar^{2}}{m}\left(\frac{\partial_{\mu} \partial^{\mu}|\psi|}{|\psi|}+\lambda|\psi|^{2}\right)
$$

and where $P^{2}=P i P i$ is the modulus of the spatial momentum.

As shown in reference [11], given the hydrodynamic Lagrangean function

$$
\begin{aligned}
& L= \frac{d S}{d t}=\frac{\partial S}{\partial t}+\frac{\partial S}{\partial q_{i}} \dot{q}_{i}=-p_{\mu} \dot{q}^{\mu} \\
&= \frac{1}{2} \frac{\sum_{n} a_{n}\left|\psi_{n}\right| \exp \left[\frac{i S_{n}}{\hbar}\right]\left(\frac{\hbar}{i} \dot{q}^{\mu} \partial_{\mu} \ln \left|\psi_{n}\right|+L_{n}\right)}{\sum_{n} a_{n}\left|\psi_{n}\right| \exp \left[\frac{i S_{n}}{\hbar}\right]} \\
&-\frac{1}{2} \frac{\sum_{n} a^{*}{ }_{n}\left|\psi_{n}\right| \exp \left[\frac{-i S_{n}}{\hbar}\right]\left(\frac{\hbar}{i} \dot{q}^{\mu} \partial_{\mu} \ln \left|\psi_{n}\right|-L_{n}\right)}{\sum_{n} a^{*}{ }_{n}\left|\psi_{n}\right| \exp \left[\frac{-i S_{n}}{\hbar}\right]}
\end{aligned}
$$

equation (2) can be expressed by the following system of Lagrangean equations of motion

$$
\begin{aligned}
& p_{\mu}=-\frac{\partial L}{\partial \dot{q}^{\mu}} \\
& \dot{p}_{\mu}=-\frac{\partial L}{\partial q^{\mu}}
\end{aligned}
$$

that for the eigenstates read

$$
\begin{aligned}
& \dot{p}_{n_{\mu}}=-\frac{\partial L_{n}}{\partial q^{\mu}} \\
& \dot{p}_{n_{\mu}}=-\frac{\partial L_{n}}{\partial q^{\mu}}
\end{aligned}
$$

where

$$
\begin{gathered}
L_{n}=( \pm)-\frac{m c^{2}}{\gamma} \sqrt{1-\frac{V_{q u(n)}}{m c^{2}}}=( \pm)-\frac{m c^{2}}{\gamma} \\
\sqrt{1+\frac{\hbar^{2}}{m^{2} c^{2}}\left(\frac{\partial_{\mu} \partial^{\mu}\left|\psi_{n}\right|}{\left|\psi_{n}\right|}+\lambda\left|\psi_{n}\right|^{2}\right)}
\end{gathered}
$$

Generally speaking, for eigenstates, for which it holds $E=E_{n}=$ const it follows that:

$$
\begin{aligned}
& \left(\frac{E_{n}{ }^{2}}{c^{2}}-p_{n}{ }^{2}\right)=m^{2} c^{2}\left(1-\frac{V_{q u(n)}}{m c^{2}}\right) \\
& =m^{2} \gamma^{2} c^{2}\left(1-\frac{V_{q u(n)}}{m c^{2}}\right)-m^{2} \gamma^{2} \dot{q}^{2}\left(1-\frac{V_{q u(n)}}{m c^{2}}\right)
\end{aligned}
$$

from where it follows that

$$
\begin{aligned}
& E_{n}= \pm m \gamma c^{2} \sqrt{1-\frac{V_{q u(n)}}{m c^{2}}}= \pm m \gamma c^{2} \\
& \sqrt{1+\frac{\hbar^{2}}{m^{2} c^{2}}\left(\frac{\partial_{\mu} \partial^{\mu}\left|\psi_{n}\right|}{\left|\psi_{n}\right|}+\lambda\left|\psi_{n}\right|^{2}\right)}
\end{aligned}
$$
that

(where the minus sign stands for antiparticles) and, by using (17),

$$
p_{n \mu}= \pm m \gamma \dot{q}_{\mu} \sqrt{1-\frac{V_{q u(n)}}{m c^{2}}}=\frac{E_{n}}{c^{2}} \dot{q}_{\mu}
$$

Following the hydrodynamic protocol [11], the eigenstates are represented by the stationary solutions of the hydrodynamic equations of motion obtained by deriving $p_{i}(\dot{q}, q)$ from (14) and then inserting it into (15) that leads to

$$
\begin{aligned}
\frac{d p_{n \mu}}{d s} & =-\frac{\gamma}{c} \frac{\partial L_{n}}{\partial q^{\mu}}= \pm \frac{d}{d s}\left(m c u_{\mu}\left(\sqrt{1-\frac{V_{q u)(n)}}{m c^{2}}}\right)\right) \\
& = \pm m c \frac{\partial}{\partial q^{\mu}} \sqrt{1-\frac{V_{q u(n)}}{m c^{2}}}
\end{aligned}
$$

where

$u_{i}=\frac{\tilde{a}}{c} \dot{q}_{i}$

and to

$$
\begin{aligned}
& \pm m c \sqrt{1-\frac{V_{q u(n)}}{m c^{2}} \frac{d u_{\mu}}{d s}}=( \pm)-m c u_{\mu} \frac{d}{d s}\left(\sqrt{1-\frac{V_{q u(n)}}{m c^{2}}}\right) \\
& \pm m c \frac{\partial}{\partial q^{\mu}}\left(\sqrt{1-\frac{V_{q u(n)}}{m c^{2}}}\right)=-\frac{\gamma}{c} \frac{\partial \mathrm{T}_{n \mu^{v}}}{\partial q^{v}}
\end{aligned}
$$

where, for eigenstates, the quantum energy-impulse tensor (QEIT) $\mathrm{T}_{n \mu}{ }^{v}$ reads $[11,12]$,

$$
\mathrm{T}_{n \mu}{ }^{v}=\left(\dot{q}_{\mu} \frac{\partial L_{n}}{\partial \dot{q}_{v}}-L_{n} \delta_{\mu}{ }^{v}\right)= \pm \frac{m c^{2}}{\gamma} \sqrt{1-\frac{V_{q u(n)}}{m c^{2}}}\left(u u u^{v}-\delta_{\mu}{ }^{v}\right)
$$

leading to the quantum energy impulse tensor density (QIETD) $[11,12]$,

$$
\begin{aligned}
& T_{n \mu}{ }^{v}=\dot{q}_{\mu} \frac{\partial \boldsymbol{L}_{n n}}{\partial \dot{q}_{\nu}}-\boldsymbol{L}_{n n} \delta_{\mu}{ }^{v}=\left|\psi_{n}\right|^{2} \\
& \left(\dot{q}_{\mu} \frac{\partial L_{n}}{\partial \dot{q}_{v}}-L_{n} \delta_{\mu}{ }^{v}\right)=\left|\psi_{n}\right|^{2} \mathrm{~T}_{n \mu}{ }^{v}
\end{aligned}
$$

where $\boldsymbol{L}=|\psi|^{2} \mathrm{~L}$ is the (hydrodynamic) Lagrangian density and $L$ is the hydrodynamic Lagrangian function. Moreover, by using the identity

$$
S_{n}=\frac{\hbar}{2 i} \ln \left[\frac{\psi_{n}}{\psi_{n} *}\right]
$$

The QIETD (23) can be written as a function of the wave function as following: 


$$
\begin{aligned}
T_{n \mu}{ }^{v}= & \pm \frac{m\left|\psi_{n}\right|^{2} c^{2}}{\gamma} \sqrt{1-\frac{V_{q u(n)}}{m c^{2}}}\left(u_{\mu} u^{v}-\delta_{\mu}{ }^{v}\right) \\
& = \pm|\psi|^{2} c^{2}\left(\frac{\partial S_{n}}{\partial t}\right)^{-1}\left(p_{\mu} p^{v}-p_{\alpha} p^{\alpha} \delta_{\mu}{ }^{v}\right) \\
& = \pm m\left|\psi_{n}\right|^{2} c^{2}\left(\frac{\frac{\hbar}{2 i m^{2} c^{2}} \partial \ln \left[\frac{\psi_{n}}{\psi^{*}{ }_{n}}\right]}{\partial t}\right)^{-1} \\
& \left(\left(\frac{\hbar}{2 m c}\right)^{2} \frac{\partial \ln \left[\frac{\psi_{n}}{\psi^{*}{ }_{n}}\right]}{\partial q^{\mu}} \frac{\partial \ln \left[\frac{\psi_{n}}{\psi^{*}{ }_{n}}\right]}{\partial q_{\nu}}+\left(1-\frac{V_{q u(n)}}{m c^{2}}\right) \delta_{\mu}{ }^{v}\right)
\end{aligned}
$$

\section{Charged field}

In the case of a charged boson field, equations (1-3) read, respectively,

$D_{\mu} D_{\mu} \psi=-\psi\left(\frac{m^{2} c^{2}}{\hbar^{2}}+\lambda|\psi|^{2}\right)$

where $D_{\mu}=\partial_{\mu}-\frac{e}{i \hbar} A_{\mu}$

$\left(\frac{\partial S_{(q, t)}}{\partial q^{\mu}}+e A^{\mu}\right)\left(\frac{\partial S_{(q, t)}}{\partial q_{\mu}}+e A_{\mu}\right)$

$=m^{2} c^{2}+\hbar^{2}\left(\frac{\partial_{\mu} \partial^{\mu}|\psi|}{|\psi|}+\lambda|\psi|^{2}\right)$

$\frac{\partial J_{i}}{\partial q_{i}}=0$

where the 4-current $J_{i}$ reads

$$
\begin{aligned}
J_{\mu}= & \left(c \rho,-j_{i}\right)=\frac{\hbar}{2 i m}\left(\psi^{*}\left(\partial_{\mu}-\frac{e}{i \hbar} A_{\mu}\right) \psi-\psi\left(\partial_{\mu}+\frac{e}{i \hbar} A_{\mu}\right) \psi^{*}\right) \\
= & \frac{\hbar}{2 i m}\left[\left(\psi^{*} \partial_{\mu} \psi-\psi \partial_{\mu} \psi^{*}\right)-\frac{2 e}{i \hbar} A_{\mu} \psi \psi^{*}\right] \\
& =-\frac{|\psi|^{2}}{m}\left[p_{\mu}-e A_{\mu}\right]=-\frac{|\psi|^{2}}{m} \pi_{\mu}
\end{aligned}
$$

and

$-\frac{\partial S}{\partial q_{\mu}}=p_{\mu}=\pi_{\mu}+e A_{\mu}=\left(\frac{E}{c},-p_{i}\right)$

(where $\pi_{\mu}$ is the mechanical momentum) $[11,13]$ and where

$\rho=-\frac{|\psi|^{2}}{m c^{2}}\left[\frac{\partial S}{\partial t}+e \phi\right]$.

Moreover, analogously to (9,17-19), from (27) it follows that

$$
\begin{aligned}
\pi_{n_{\mu}} & =\frac{1}{c^{2}}\left[-\frac{\partial S_{n}}{\partial t}-e \phi\right] \dot{q}_{\mu} \\
& =\frac{E_{n}-e \phi}{c^{2}} \dot{q}_{\mu}=p_{n_{\mu}}-e A_{\mu}
\end{aligned}
$$

that leads to

$$
\begin{aligned}
& E_{n}-e \phi= \pm m \gamma c^{2} \sqrt{1-\frac{V_{q u(n)}}{m c^{2}}}= \\
& \pm m \gamma c^{2} \sqrt{1+\frac{\hbar^{2}}{m^{2} c^{2}}\left(\frac{\partial_{\mu} \partial^{\mu}\left|\psi_{n}\right|}{\left|\psi_{n}\right|}+\lambda\left|\psi_{n}\right|^{2}\right)}
\end{aligned}
$$

to

$$
p_{n \mu}-e A_{\mu}= \pm m \gamma \dot{q}_{\mu} \sqrt{1-\frac{V_{q u(n)}}{m c^{2}}}=\frac{E_{n}}{c^{2}} \dot{q}_{\mu}
$$

and to

$$
\begin{aligned}
L_{n} & =\frac{d S_{n}}{d t}=\frac{\partial S_{n}}{\partial t}+\frac{\partial S_{n}}{\partial q_{i}} \dot{q}_{i}=-p_{n \mu} \dot{q}^{\mu}=-\left(\frac{1}{c^{2}} \frac{\partial S_{n}}{\partial t}+\frac{e}{c^{2}} \phi\right)^{-1} p_{n \mu}\left(p_{n}{ }^{\mu}-e A^{\mu}\right) \\
& =\rho_{n}{ }^{-1} p_{n \mu} J_{n}{ }^{\mu} \\
& =( \pm)\left(-\frac{m c^{2}}{\gamma} \sqrt{1+\frac{\hbar^{2}}{m^{2} c^{2}}\left(\frac{\partial_{\mu} \partial^{\mu}\left|\psi_{n}\right|}{\left|\psi_{n}\right|}+\lambda\left|\psi_{n}\right|^{2}\right)}-e A_{\mu} \dot{q}^{\mu}\right)
\end{aligned}
$$

that, by using (24), as a function of $\psi$ and $A^{i}$, reads

$$
\begin{aligned}
L_{n} & =\frac{d S_{n}}{d t}=\frac{\partial S_{n}}{\partial t}+\frac{\partial S_{n}}{\partial q_{i}} \dot{q}_{i}=-p_{n \mu} \dot{q}^{\mu}=-\left(\frac{1}{c^{2}} \frac{\partial S_{n}}{\partial t}+\frac{e}{c^{2}} \phi\right)^{-1} p_{n \mu}\left(p_{n}{ }^{\mu}-e A^{\mu}\right) \\
& =\rho_{n}{ }^{-1} p_{n \mu} J_{n}{ }^{\mu} \\
& =( \pm)\left(-\frac{m c^{2}}{\gamma} \sqrt{1+\frac{\hbar^{2}}{m^{2} c^{2}}\left(\frac{\partial_{\mu} \partial^{\mu}\left|\psi_{n}\right|}{\left|\psi_{n}\right|}+\lambda\left|\psi_{n}\right|^{2}\right)}-e A_{\mu} \dot{q}^{\mu}\right)
\end{aligned}
$$

Moreover, with the help of $(24,29,32-34)$ it follows that

$$
\begin{aligned}
& T_{n \mu}{ }^{\nu}=( \pm)-\left|\psi_{n}\right|^{2}\left(p_{n \mu} \dot{q}^{\nu}-p_{n \alpha} \dot{q}^{\alpha} \delta_{\mu}{ }^{\nu}\right)=-\left(\frac{1}{m c^{2}}\left(\frac{\partial S_{n}}{\partial t}+e \phi\right)\right)^{-1}\left(p_{n \mu} J_{n}{ }^{\nu}-p_{n \alpha} J_{n}{ }^{\alpha} \delta_{\mu}{ }^{\nu}\right) \\
& =( \pm)-\left(m \rho_{n}\right)^{-1}\left[\left(J_{n \mu} J_{n}{ }^{v}-J_{n \alpha} J_{n}{ }^{\alpha} \delta_{\mu}{ }^{\nu}\right)-\frac{e|\psi|^{2}}{m}\left(A_{\mu} J_{n}{ }^{\nu}-A_{\alpha} J_{n}{ }^{\alpha} \delta_{\mu}{ }^{\nu}\right)\right]
\end{aligned}
$$

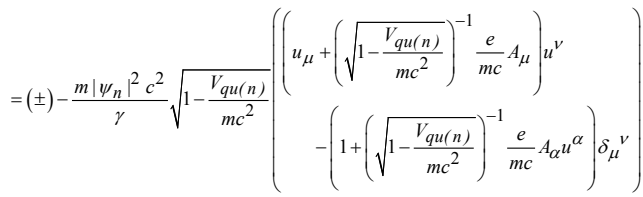

that, by using $(24,29,34)$ we can express as a function of the wave function as

$$
\begin{aligned}
& T_{\mu}{ }^{\nu}=-\left|\psi_{n}\right|^{2} c^{2}\left[-\frac{\partial S}{\partial t}+e \phi\right]^{-1}\left(p_{n \mu}\left(p_{n}{ }^{\nu}-e A^{\nu}\right)-p_{n \alpha}\left(p_{n}{ }^{\alpha}-e A^{\alpha}\right) \delta_{\mu}{ }^{\nu}\right) \\
& =\left|\psi_{n}\right|^{2} \frac{\hbar c^{2}}{2 i}\left(\frac{\partial}{\partial t} \ln \left[\frac{\psi_{n}}{\psi_{n}{ }^{*}}\right]-\frac{2 i e}{\hbar} \phi\right)^{-1}\left(\begin{array}{l}
\left(\frac{\partial \ln \left[\frac{\psi_{n}}{\psi_{n}{ }^{*}}\right]}{\partial q^{\mu}}-\frac{2 i e}{\hbar} A_{\mu}\right) \frac{\partial \ln \left[\frac{\psi_{n}}{\psi_{n}{ }^{*}}\right]}{\partial q_{V}} \\
-\left(\frac{\partial \ln \left[\frac{\psi_{n}}{\psi_{n}{ }^{*}}\right]}{\partial q^{\alpha}}-\frac{2 i e}{\hbar} A_{\alpha}\right) \frac{\partial \ln \left[\frac{\psi_{n}}{\left.\psi_{n}{ }^{*}\right]}\right.}{\partial q_{\alpha}} \delta_{\mu}{ }^{v}
\end{array}\right)
\end{aligned}
$$

The above equations are coupled to the Maxwell one

$F_{\mu v ; v}=-4 \pi J^{\mu}$

(where $J_{\mu}=\frac{\hbar}{2 i m}\left(\psi^{*} D_{\mu} \psi-\psi D_{\mu} \psi^{*}\right.$ ) is the current of the charged particles) where [14]

$$
F_{\mu v}=\left(A_{v ; \mu}-A_{\mu ; v}\right)=\left(\partial_{\mu} A_{v}-\partial_{v} A_{\mu}\right)
$$

and where 


$$
A^{i}=\left(\frac{\phi}{c},-A_{i}\right)
$$

is the potential 4-vector,

\section{Non-Euclidean Generalization}

The quartic self-interaction is introduced in the KGE in order to describe the states of charged $( \pm 1)$ bosons (e.g., mesons) [15]. The importance of having the hydrodynamic description of bosons [11] lies in the fact that it allows to derive its quantum energy-impulse tensor that can couple them to the Einstein quantum-gravitational equation [11].

The generalization of the quantum hydrodynamic formalism to the non-Euclidean space-time can be obtained by using the General Physics Covariance postulate $[11,16]$. By using it, it is possible to derive the non-Euclidean expression of the hydrodynamic model of the KGE

$$
\left(\partial^{\mu} \psi\right)_{; \mu}=\frac{1}{\sqrt{-g}} \partial \mu\left(\sqrt{-g} g \mu \nu \partial_{\nu} \psi\right)=-\psi\left(\frac{m^{2} c^{2}}{\hbar^{2}}+\lambda|\psi|^{2}\right)
$$

Equations (2-3) in a non- Euclidean space read, respectively,

$$
\begin{aligned}
& g^{\mu v} \frac{\partial S_{(q, t)}}{\partial q^{\mu}} \frac{\partial S_{(q, t)}}{\partial q^{v}}+m V_{q u}-m^{2} c^{2}=0 \\
& \frac{1}{\sqrt{-g}} \frac{\partial}{\partial q^{\mu}} \sqrt{-g}\left(g^{\mu v}|\psi|^{2} \frac{\partial S}{\partial q^{v}}\right)=0
\end{aligned}
$$

where

$$
V_{q u}=-\frac{\hbar^{2}}{m}\left(\frac{1}{|\psi| \sqrt{-g}} \partial_{\mu} \sqrt{-g}\left(g^{\mu v} \partial_{v}|\psi|\right)+\lambda|\psi|^{2}\right)
$$

Moreover, by using the definition of the Lagrangean function

$$
L_{n}=\frac{d S_{n}}{d t}=-g_{\mu v} p_{n}^{v} \dot{q}^{\mu}
$$

the covariant form of the motion equations (14-15) reads

$$
\begin{aligned}
& D_{t} p_{n_{\mu}}=-\frac{\partial L_{n}}{\partial q^{\mu}} \\
& D_{t} p_{n_{\mu}}=-\frac{\partial L_{n}}{\partial q^{\mu}}
\end{aligned}
$$

where

$$
D_{t} p_{\mu}=\dot{p}_{\mu}-\Gamma_{\mu \lambda}^{v} p_{v} \dot{q}^{\lambda}
$$

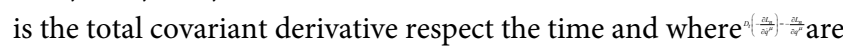
the Christoffel symbols.

Equations (45-46) leads to the motion equation

$$
D_{t}\left(-\frac{\partial L_{n}}{\partial \dot{q}^{\mu}}\right)=-\frac{\partial L_{n}}{\partial q^{\mu}}
$$

where, $L_{n}$ reads

$$
\begin{aligned}
& L_{n}=( \pm)-\frac{m c^{2}}{\gamma} \sqrt{1-\frac{V_{q u(n)}}{m c^{2}}}= \\
& ( \pm)-m c^{2} \sqrt{\frac{g_{\mu \nu} \dot{q}^{v} \dot{q}^{\mu}}{c^{2}}} \sqrt{1-\frac{V_{q u(n)}}{m c^{2}}}
\end{aligned}
$$

From (48) it follows that the motion equation reads

$$
\begin{aligned}
& \frac{d u_{\mu}}{d t}-\frac{1}{2} \frac{c}{\gamma} \frac{\partial g_{\lambda \kappa}}{\partial q^{\mu}} u^{\lambda} u^{\kappa}= \\
& \frac{c}{\gamma} \frac{\partial \ln \sqrt{1-\frac{V_{q u(n)}}{m c^{2}}}}{\partial q^{\mu}}+\frac{c}{\gamma} \frac{\partial \ln \gamma}{\partial q^{\mu}}-u_{\mu} \frac{d}{d t} \ln \sqrt{1-\frac{V_{q u}}{m c^{2}}}
\end{aligned}
$$

where the stationary condition $\frac{d u_{\mu}}{d t}=0$, that determines the balance between the "force" of gravity and that one of the quantum potential, leads to the stationary equation for the Eigen states

$$
\begin{aligned}
& \frac{1}{2} \frac{c}{\gamma} \frac{\partial g_{\lambda \kappa}}{\partial q^{\mu}} u^{\lambda} u^{\kappa}+\frac{c}{\gamma} \frac{\partial \ln \gamma}{\partial q^{\mu}}= \\
& -\frac{c}{\gamma} \frac{\partial \ln \sqrt{1-\frac{V_{q u(n)}}{m c^{2}}}}{\partial q^{\mu}}+u \mu \frac{d}{d t} \ln \sqrt{1-\frac{V_{q u}}{m c^{2}}} \\
& \text { where } \frac{1}{g}=\left|g_{v \mu}\right|=-J_{a c}{ }^{2} \text {, where } J_{a c} \text { is Jacobean of the }
\end{aligned}
$$

transformation of the Galilean co-ordinates to non-Euclidean ones and where $g_{i i}$ is the metric tensor defined by the quantum gravitational equation [11]

$$
R_{\mu v}-\frac{1}{2} g_{\mu v} R_{\alpha}^{\alpha}-\Lambda g_{\mu v}=\frac{8 \pi G}{c^{4}} T_{\mu v}
$$

where the quantum energy impulse tensor density reads

$$
\begin{aligned}
& T_{(n) \mu v}= \pm\left|\psi_{n}\right|^{2} m c^{2} \sqrt{\frac{g_{\mu v} \dot{q}^{v} \dot{q}^{\mu}}{c^{2}}} \\
& \sqrt{1-\frac{V_{q u(n)}}{m c^{2}}}\left(u_{\mu} u_{v}-g_{\alpha \beta} u^{\alpha} u^{\beta} g_{\mu v}\right)
\end{aligned}
$$

and where the cosmological energy-impulse density $\Lambda$ [11], for Eigen states, reads

$$
\Lambda=\frac{8 \pi G}{c^{4}}\left|\psi_{n}\right|^{2} L_{0}=\frac{8 \pi G}{c^{4}} \frac{m\left|\psi_{n}\right|^{2} c^{2}}{\gamma}
$$

where, for scalar uncharged particles leads to

$$
L_{0}=\lim _{\hbar \rightarrow 0} L=( \pm)-\frac{m c^{2}}{\gamma}
$$

Finally, it is worth noting that, as a function of the quantum field, the quantum energy impulse tensor density reads

$$
\begin{aligned}
T_{\mu \nu}=T_{\mu}^{\alpha} g_{\alpha \nu}= & |\psi|^{2} m^{2} c^{4}\left(\frac{\partial S}{\partial t}\right)^{-1}\left(\frac{p_{\mu} p_{v}}{m^{2} c^{2}}-\left(1-\frac{V_{q u}}{m c^{2}}\right) g_{\mu \nu}\right) \\
& =\frac{m|\psi|^{2} c^{2}}{\gamma}\left(\frac{1}{m \gamma c^{2}} \frac{\partial S}{\partial t}\right)^{-1}\left(u_{\mu} u_{v}-\left(1-\frac{V_{q u}}{m c^{2}}\right) g_{\mu \nu}\right) \\
& =m|\psi|^{2} c^{2}\left(\frac{\frac{\hbar}{2 i m^{2} c^{2}} \partial \ln \left[\frac{\psi}{\psi^{*}}\right]}{\partial t}\right)^{-1} \\
& \left(\left(\frac{\hbar}{2 m c}\right)^{2} \frac{\partial \ln \left[\frac{\psi}{\psi^{*}}\right]}{\partial q^{\mu}} \frac{\partial \ln \left[\frac{\psi}{\psi^{*}}\right]}{\partial q^{v}}+\left(1-\frac{V_{q u}}{m c^{2}}\right) g_{\mu v}\right)
\end{aligned}
$$


Citation: Chiarelli P (2017) The Hydrodynamic Representation of the Klein-Gordon Equation with Self-Interacting Field. J Astrophys Aerospace Technol 5: 148. doi:10.4172/2329-6542.1000148

Page 5 of 5

\section{Charged boson in non-Euclidean space-time}

The KGE in non-Euclidean space-time for electromagnetic charged boson

$$
\frac{1}{\sqrt{-g}} D^{\mu}\left(\sqrt{-g} D_{\mu} \psi\right)-\frac{A_{\mu} \partial^{\mu} \sqrt{-g}}{\sqrt{-g}}=-\psi\left(\frac{m^{2} c^{2}}{\hbar^{2}}+\lambda|\psi|^{2}\right)
$$

leads to the hydrodynamic system of equations

$$
\begin{aligned}
& \frac{1}{\sqrt{-g}} \frac{\partial}{\partial q^{\mu}} \sqrt{-g}\left(g^{\mu v}|\psi|^{2}\left(\frac{\partial S}{\partial q^{v}}+e A_{\mu}\right)\right)=0 \\
& V_{q u}=-\frac{\hbar^{2}}{m}\left(\frac{1}{|\psi| \sqrt{-g}} \partial_{\mu} \sqrt{-g}\left(g^{\mu v} \partial_{v}|\psi|\right)+\lambda|\psi|^{2}\right)
\end{aligned}
$$

where

$$
V_{q u}=-\frac{\hbar^{2}}{m}\left(\frac{1}{|\psi| \sqrt{-g}} \partial \mu \sqrt{-g}\left(g^{\mu \nu} \partial_{\nu}|\psi|\right)+\lambda|\psi|^{2}\right)
$$

Moreover, the Lagrangean motion equations read

$$
\begin{aligned}
& p_{n_{\mu}}=-\frac{\partial L_{n}}{\partial \dot{q}^{\mu}} \\
& D_{t} p_{n_{\mu}}=-\frac{\partial L_{n}}{\partial q^{\mu}}
\end{aligned}
$$

where

$$
L_{n}=( \pm)\left(\sqrt{-m c^{2} \sqrt{\frac{g_{\mu v} \dot{q}^{v} \dot{q}^{\mu}}{c^{2}}}}\left(\sqrt{1+\frac{\hbar^{2}}{m^{2} c^{2}}\left(\frac{\partial_{\mu} \partial^{\mu}\left|\psi_{n}\right|}{\left|\psi_{n}\right|}+\lambda\left|\psi_{n}\right|^{2}\right)}-g_{\mu \nu} e A^{v} \dot{q}^{\mu}\right)\right.
$$

and to the QIETD

$$
\begin{aligned}
& T_{n \mu}{ }^{v}=( \pm)-m\left|\psi_{n}\right|^{2} c^{2} \sqrt{\frac{g_{\mu \nu} \dot{q}^{v} \dot{q}^{\mu}}{c^{2}}} \sqrt{1-\frac{V_{q u(n)}}{m c^{2}}} \\
& \left(\begin{array}{l}
\left(u_{\mu}+\left(\sqrt{1-\frac{V_{q u(n)}}{m c^{2}}}\right)^{-1} \frac{e}{m c} A_{\mu}\right) u^{v} \\
\left.-\left(1+\left(\sqrt{1-\frac{V_{q u(n)}}{m c^{2}}}\right)^{-1} \frac{e}{m c} g_{\alpha \beta} A^{\beta} u^{\alpha}\right) \delta_{\mu}{ }^{v}\right)
\end{array}\right.
\end{aligned}
$$

\section{Conclusion}

The hydrodynamic approach allows obtaining the quantum energy-impulse tensor density as a function the field of the particle.

The biunique correspondence between the standard quantum mechanics and the hydrodynamic representation [1-6,17] warrants that the quantum energy-impulse tensor density can be independently defined by the used formalism.

In this work the quantum energy-impulse tensor, for massive bosons described by a KGE with self-interacting field is derived for defining the coupling with the quantum gravitational equation.

\section{References}

1. Madelung E (1926) Quantum theory in hydro-dynamical form Z. Phys 40: 322-326

2. Guvenis S (2014) Hydrodynamische formulierung der relativischen Quantenmechanik. The Gen Sci J.

3. Bialyniki BI, Cieplak M, Kaminski J (1992) Theory of quanta. Oxford University press, New York, USA. pp. 87-111.

4. Jánossy L (1962) Zum hydrodynamischen Modell der Quantenmechanik Z Phys 169: 79-89.

5. Chiarelli P (2015) Theoretical derivation of the cosmological constant in the framework of the hydrodynamic model of quantum gravity: The solution of the quantum vacuum catastrophe? Galaxies.

6. Weiner JH, Askar A (1971) Particle method for the numerical solution of the time-dependent Schrödinger equation J Chem Phys 54: 34-35.

7. Koide T, Kodama T (2014) Stochastic variational method as quantization scheme: Field quantization of complex Klein-Gordon equation. Progress of Theoretical and Experimental Physics p. 1: 2.

8. Chiarelli P (2016) The Planck law for particles with rest mass. Quantum Matt 5: 748-751.

9. Hiley BJ (2010) The Bohm approach re-assessed, pre-print.

10. Hiley BJ, Callaghan RE (2010) The Clifford Algebra approach to quantum mechanics A: The Schrödinger and Pauli particles p.1-29.

11. Chiarelli $P$ (2017) The quantum-gravity equation derived from the minimum action principle. Classical and Quantum Gravity.

12. Chiarelli $P$ (2016) The quantum lowest limit to the black hole mass. Phys Sci Int J 9: 1-25.

13. Chiarelli $P$ (2016) The CPT-Ricci scalar curvature symmetry in quantum electro-gravity. Int J Sci 5: 36-58.

14. Landau LD, Lifsits EM (1976) Course of theoretical physics. (Italian edition), Mir Mosca, Editori Riuniti (eds), 2: 309-335.

15. Bellac ML (1991) Quantum and statistical field theory. Oxford Science Publication, Oxford, London. pp. 315-337.

16. Ashtekar A (2011) Introduction to loop quantum gravity.

17. Tsekov R (2015) Bohemian mechanics versus Madelung quantum hydrodynamics. 